\title{
2D-FEM Modeling of an Electromagnetic Energy Recovery Damper for Vehicle Applications
}

\author{
Mustapha Zaouia, Hakim Denoun, Nabil Benyahia, Mustapha Bouheraoua, and \\ Nacereddine Benamrouche \\ Laboratoire des Technologies Avancées en Génie Electrique, Département d'Electrotechnique, \\ FGEI, Université Mouloud Mammeri de Tizi-Ouzou, B.P 17 RP Tizi-Ouzou 15000, Algeria, \\ zbmust@yahoo.fr
}

\begin{abstract}
The objective of this paper is to develop a 2D Finite Elements (FEM) model to study and analyse an Electromagnetic Energy Recovery Damper (EERD) for road vehicles to quantify the electrical power output for various cycles of vibrations created by roads irregularities. The EERD transforms the kinetic energy due to vibrations to electrical energy. The developed model is based on the governing magnetic field equations expressed in terms of magnetic vector potential solved using the nodal-based finite element method and sequentially coupling between the electromagnetic and the mechanical equations obtained after modelling a quarter of the vehicle system. The coupling is carried out firstly through the magnetic force, and secondly by the modified flux distribution due to the moving part displacements simulated by the Macro-Element method. The cycles of vibrations are transmitted to the device as vertical displacements. The obtained results are mainly the electrical power output, the deflection, the magnetic force and the induced electromotive force, for considered cycles of vibrations.
\end{abstract}

Keywords: Energy Recovery Damper, Electrical Power, vehicles, 2D Finite Elements, Macro-Element.

\section{Introduction}

In recent years, many problems have been raised by extensive use of fossil energies leading to their high cost and may be their depletion. Therefore, alternative energies are essential, particularly for automotive applications. One of the most promising advanced technologies is hybrid and electric vehicles but until now their efficiencies remain weak; this requires the recovery of all the possible kinetic energies among which those of vibrations and to transform them to electrical energy in order to improve the efficiency of the vehicles. To achieve this, electromagnetic dampers are used principally in order to improve the comfort of the vehicles by compensating the inconvenience caused by road irregularities. In fact, their development is in constant evolution. Several authors have proposed different types. In [1] and [2], these systems are based on rotary actuators; however the use of these devices requires motion converters which have many disadvantages. Now, research is focusing on the development of electromagnetic dampers using linear tubular topologies since they do not require a motion converter [3-7].

In this work our objective is the presentation and the analysis of an electromagnetic damper named Energy Recovery Damper (EERD) which operates as a generator. The damper transforms the kinetic energy of vibrations caused by road irregularities into electrical energy to improve the efficiency of electrical or hybrid vehicles. This energy will be used to supply the electrical components of these vehicles or to restore the electrical energy to their batteries. The (EERD) is a permanent magnet (PM) linear machine with tubular form. The working magnetic field is created using permanent magnets mounted on the translator of the generator. The magnetic flux is changed by varying the magnetic field across the coil by moving the magnet relative to the coil. The permanent magnets are cylindrical with axial magnetization

Received: December $11^{\text {st }}$, 2013. Accepted: February $24^{\text {th }}, 2014$ 
polarized length-wise, and are placed between the ferromagnetic cylindrical pole shoes which are used to guide the magnetic flux over the air gap into the stator. The magnets are placed on the mover support in such a way to make the magnetic polarities on the surface of the mover alternate in the axial direction.

The study and analyse of the EERD are carried out by the application of an electromagnetic - mechanical coupled model. This model is developed and implemented under Matlab environment. The magnetic field equation expressed in terms of Magnetic Vector Potential (MVP) in the case of two-dimensional cylindrical coordinates governing the electromagnetic device is solved using the Finite Element Method (FEM). The mechanical equation is given after modelling a quarter of vehicle [7], [10] and solved numerically to get the displacements, the deflections and the velocities.

The electromagnetic and the mechanical equations are sequentially coupled through the magnetic force and the modified flux distribution due to the moving part displacement. The high accuracy required for taking into account the moving parts has led us to adopt the MacroElement (ME) method [11],[12]. The ME technique is based on the analytical solution of Laplace equation in the unmeshed air-gap region which separates the meshed fixed and moving regions. The unmeshed air gap appears as a multi-nodes finite element, and therefore while the moving region displaces, only the node coordinates of the air-gap element are moved which permits to maintain unchanged the mesh topology at each displacement. Without any constrains on the mesh topology, the major advantages of the ME technique are the possibility to manage variable displacement steps which are obtained through the mechanical equation, allowing to accurately calculate the magnetic force.

The geometrical characteristics of the EERD are given by [8] and [9]. After modelling and application of the developed electromagnetic-mechanical model to study and analyse the EERD, the obtained results are mainly the electrical generated output power, the deflection, the magnetic force, the electromotive force, and the current. These results are obtained for various cycles of vibrations injected as vertical displacements.

\section{Description of the EERD-Single Wheel System}

The mechanical system represents a quarter of the road vehicle [7], [10] (Figure1) is composed of two distinct masses. The sprung mass represents the mass of car chassis, and the unsprung mass represents the mass of the wheel with the suspension and brake equipment. The road position reference represents a cycle of vibration. The mechanical parameters are presented in Table 1.The electromagnetic damper is a permanent magnet machine operating as a generator. The permanent magnets used are of $\mathrm{NdFeB}$ type axially magnetized. The geometrical and the study domain of the electromagnetic damper are presented in Figure 2 [1], [2]. Its dimensions and characteristics are detailed in Table 2.

Table 1. Mechanical parameters

\begin{tabular}{|c|c|}
\hline Quantity / Symbol & Value \\
\hline Sprung mass $\quad\left(m_{s}\right)$ & $290 \mathrm{~kg}$ \\
\hline Unsprung mass $\left(m_{u}\right)$ & $59 \mathrm{~kg}$ \\
\hline Spring stiffness $\left(k_{s}\right)$ & $16000 \mathrm{~N} / \mathrm{m}$ \\
\hline Tyre stiffness $\quad\left(k_{u}\right)$ & $190000 \mathrm{~N} / \mathrm{m}$ \\
\hline Damper coefficient $\left(b_{s}\right)$ & $1000 \mathrm{~N} / \mathrm{m} / \mathrm{s}$ \\
\hline
\end{tabular}




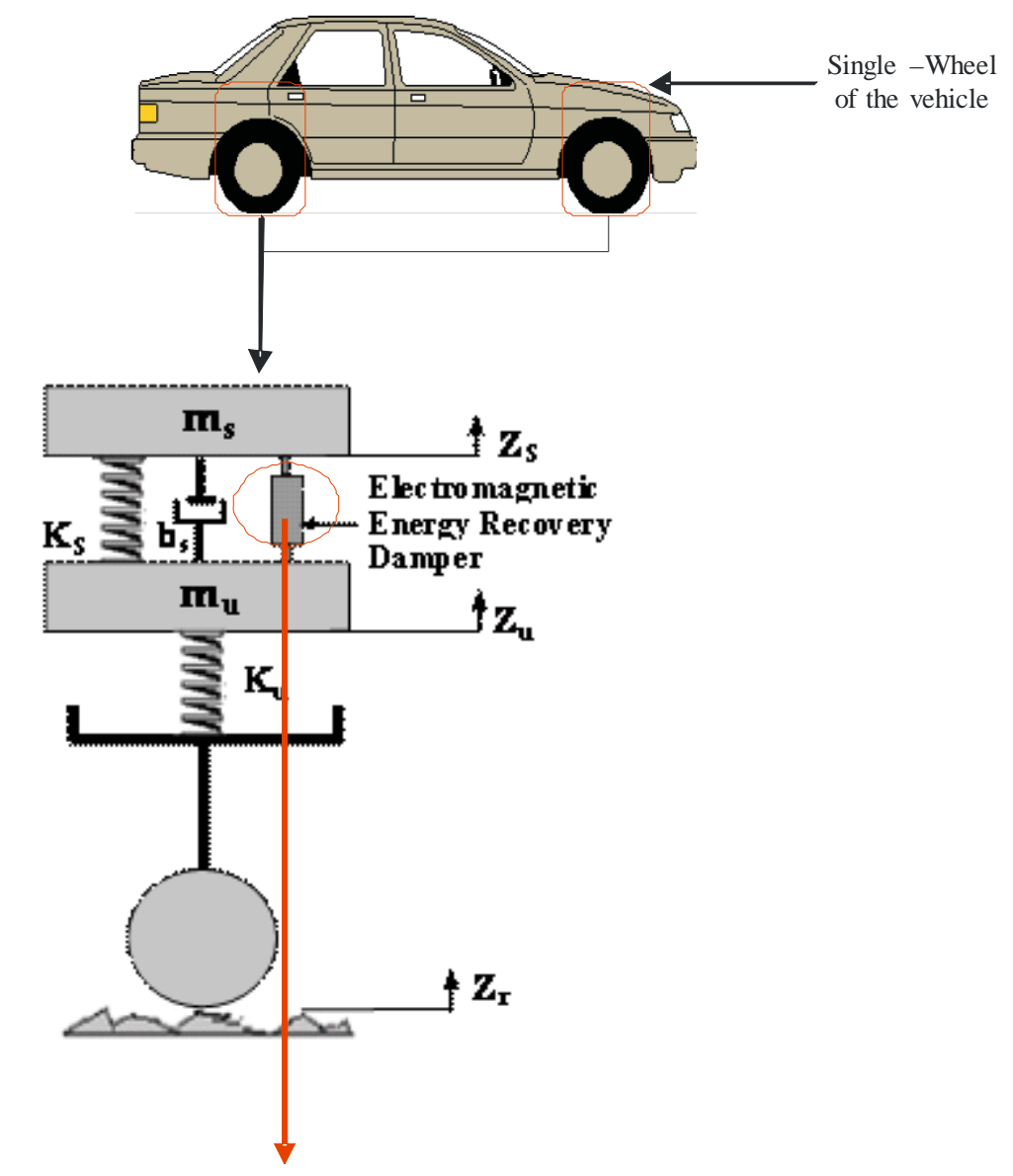

Figure 1. Model of an electromagnetic single-wheel damper

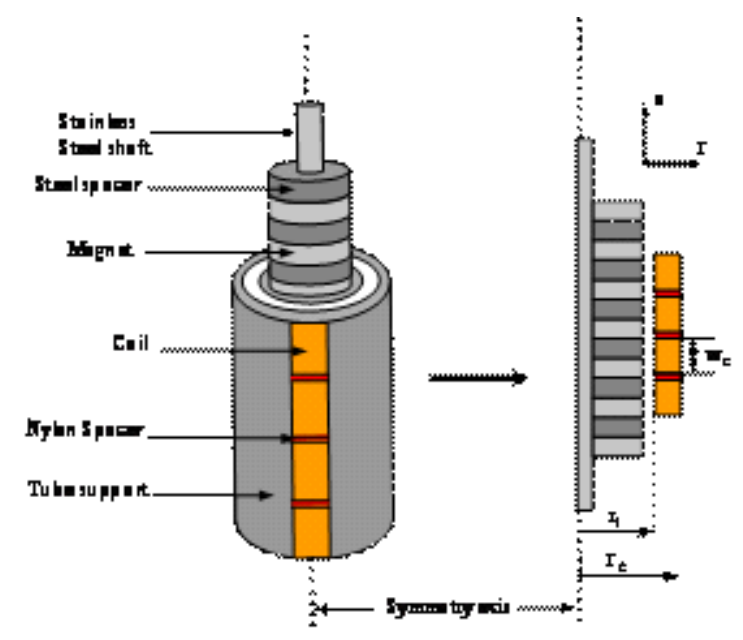

Figure 2. Geometry (a) and study domain (b) of the Electromagnetic Energy Recovery Damper 
Table 2. Dimensions and characteristics of the EERD

\begin{tabular}{|l|c|}
\hline \hline \multicolumn{1}{|c|}{ Quantity / Symbol } & Value \\
\hline Diameter of Stainless steel shaft & $0.02 \mathrm{~m}$ \\
\hline Diameter of Steel spacer & $0.1 \mathrm{~m}$ \\
\hline Diameter of permanent magnet & $0.1 \mathrm{~m}$ \\
\hline Permanent magnet width & $0.025 \mathrm{~m}$ \\
\hline Steel width & $0.025 \mathrm{~m}$ \\
\hline Inter. Diameter of coil & $0.1 \mathrm{~m}$ \\
\hline Outer diameter of coil & $0.155 \mathrm{~m}$ \\
\hline Inter. Diameter of tube support & $0.155 \mathrm{~m}$ \\
\hline Inter. Diameter of tube support & $0.180 \mathrm{~m}$ \\
\hline Air gap & $0.005 \mathrm{~m}$ \\
\hline Coil width (w $\left.\mathrm{w}_{\mathrm{c}}\right)$ & $0.05 \mathrm{~m}$ \\
\hline Number of turns of each coil $\left(\mathrm{N}_{\mathrm{a}}\right)$ & 690 \\
\hline
\end{tabular}

\section{Electromagnetic-Mechanical modelling}

A. Electromagnetic Finite Element Formulation

Starting from Maxwell's equation, neglecting the displacement current, and taking into account the magnetic nonlinear material properties, the governing magnetic field equation in terms of magnetic vector potential is given in the $2 \mathrm{D}$ cylindrical coordinates $(r, \varphi, z)$ as follows:

$$
\begin{aligned}
& \vec{\nabla} \wedge(\vec{\nabla} \wedge \vec{A})+\frac{\sigma}{r}\left(\frac{D \vec{A}}{D t}\right)=\vec{J}_{s}+v_{o}\left(\vec{\nabla} \wedge \vec{b}_{m}\right) \\
& \vec{J}_{s}=\left(\frac{N_{a}}{S_{c}} i\right) \cdot \vec{n} \\
& \frac{D \vec{A}}{D t}=-\frac{\partial \vec{A}}{\partial t}+[\vec{v} \wedge(\vec{\nabla} \wedge \vec{A})] \\
& \vec{B}=\vec{\nabla} \wedge \vec{A}
\end{aligned}
$$

where $v$ is the magnetic reluctivity of material, $v_{0}$ the vacuum reluctivity, $\vec{A}$ the magnetic vector potential, $\sigma$ the electrical conductivity, $\vec{B}$ the flux density, $\vec{b}_{m}$ remnant induction of the permanent magnet, $\vec{J}_{s}$ the exciting current density. $N_{a}$ and $S_{c}$ are respectively the number of turns and the cross-sectional area of the coil, $\vec{n}$ is the unit vector along the direction of the exciting current $i$ and $\vec{v}$ is the body velocity. Both the stator and the rotor are meshed and separated by an unmeshed air gap known as the Macro-Element. To complete the formulation of equation (1), boundary conditions are necessary especially in the ME. Since the displacement is simulated by means of Macro Element, the connection between the fixed and the moved meshes is achieved through the MVP analytical expression at each node of the ME boundary. While the movement implicitly taken in charge by the considered ME frame, the current fed expression (1) associated to the Macro-Element technique is written in space discretised form using the Galerkine finite element method as follows: 


$$
\begin{aligned}
& \iint_{\Omega} v\left(\frac{\partial \alpha_{\boldsymbol{i}}}{\partial \boldsymbol{r}} \frac{\partial \boldsymbol{A}^{*}}{\partial \boldsymbol{r}}+\frac{\partial \alpha_{\mathbf{i}}}{\partial \mathbf{z}} \frac{\partial \boldsymbol{A}^{*}}{\partial \mathbf{z}}\right) \frac{\boldsymbol{d} \boldsymbol{r d z}}{\boldsymbol{r}}+\iint_{\Omega} \alpha_{\boldsymbol{i}} \frac{\sigma}{\boldsymbol{r}} \frac{\partial \boldsymbol{A}^{*}}{\partial \boldsymbol{t}} \boldsymbol{d r} \boldsymbol{d z} \int_{\Gamma_{M E}} v_{\boldsymbol{o}}\left[\frac{\alpha_{\boldsymbol{i}}^{M E}}{\boldsymbol{r}}\left(\frac{\partial \boldsymbol{A}^{*}}{\partial \boldsymbol{n}}\right)\right] \boldsymbol{d} \Gamma_{\boldsymbol{M E}} \\
& -\int_{\Gamma \Gamma_{M E}} v\left[\frac{\alpha_{\boldsymbol{i}}}{\boldsymbol{r}}\left(\frac{\partial \boldsymbol{A}^{*}}{\partial \boldsymbol{n}}\right)\right] \boldsymbol{d} \Gamma=\iint_{\Omega_{s}}\left(\alpha_{\boldsymbol{i}} \boldsymbol{J}_{s}\right) \boldsymbol{d r d z}+\iint_{\Omega_{P M}} v_{\boldsymbol{o}} \cdot \alpha_{\boldsymbol{i}}\left(\frac{\partial \boldsymbol{b} \boldsymbol{m}_{\mathbf{z}}}{\partial \boldsymbol{r}}-\frac{\partial \boldsymbol{b} \boldsymbol{m}_{\boldsymbol{r}}}{\partial \mathbf{z}}\right) \boldsymbol{d r d z}
\end{aligned}
$$

Where, $\boldsymbol{A}^{*}=\boldsymbol{r} \boldsymbol{A}_{\varphi}$ is the modified magnetic vector potential, $b m_{z}$ and $b m r$ are respectively the axial and radial components of the remnant induction, $\Gamma_{M E}$ the MacroElement boundary and $\Gamma$ the boundary surrounding the meshed regions area $\Omega$. The conductors and permanent magnet regions are respectively $\Omega_{s}$ and $\Omega_{P M}$. The weighted function is $\alpha_{i}$.

Using the first order triangular finite element, the magnetic vector potential is given by means of the approximation functions $\alpha_{j}(r, z)$ and $\alpha_{j}^{M E}(r, z)$ for each finite element $j$ respectively of the meshed node regions and the unmeshed Macro-element boundary nodes:

$$
\boldsymbol{A}^{*}(\boldsymbol{r}, \mathbf{z})= \begin{cases}\sum_{\boldsymbol{j}=1}^{\boldsymbol{n}} \alpha_{\boldsymbol{j}}(\boldsymbol{r}, \mathbf{z}) \boldsymbol{A}_{\boldsymbol{j}}^{*} \quad \text { in the meshed regions } \\ \sum_{\boldsymbol{j}=1}^{M E} \alpha_{\boldsymbol{j}}^{M E}(\boldsymbol{r}, \mathbf{z}) \boldsymbol{A}_{\boldsymbol{j}}^{*} \quad \text { in the Macro - Element }\end{cases}
$$

With nn and $n_{t}^{M E}$ are the numbers of the meshed region nodes and Macro-Elements boundary nodes respectively.

The introduction of approximation functions of the magnetic vector potential (6) for each node, leads to the following algebraic equation system:

$$
\left([\boldsymbol{S}]+\left[\boldsymbol{S}^{\boldsymbol{M} \boldsymbol{E}}\right]\left\{\boldsymbol{A}^{*}\right\}+[\boldsymbol{K}]\left\{\frac{\partial \boldsymbol{A}^{*}}{\partial \boldsymbol{t}}\right\}=\{\boldsymbol{F}\}+\{\boldsymbol{G}\}\right.
$$

Where:

$$
\begin{aligned}
& S_{i j}=\iint_{\Omega} v\left(\vec{\nabla} \cdot \alpha_{i} \vec{\nabla} \cdot \alpha_{j}\right) \frac{d r d z}{r} \\
& F_{i}=\iint_{\Omega}\left(\alpha_{i} J_{s}\right) d r d z \\
& K_{i j}=\int_{\Omega_{s}}\left[\sigma \alpha_{i} \alpha_{j}\right] \frac{d r d z}{r} \\
& \boldsymbol{G}_{\boldsymbol{i}}=\iint_{\Omega_{\boldsymbol{P M}}} v_{0}\left(\frac{\partial \alpha_{\boldsymbol{i}}}{\partial \boldsymbol{r}} \boldsymbol{b}_{\boldsymbol{m} \mathbf{z}}-\frac{\partial \alpha_{\boldsymbol{i}}}{\partial \mathbf{z}} \boldsymbol{b}_{\boldsymbol{m} \boldsymbol{r}}\right) \boldsymbol{d} \boldsymbol{r} \boldsymbol{d} \mathbf{z}
\end{aligned}
$$

The contribution of the Macro-Element to the global stiffness matrix is considered by the term of the matrix $S_{i j}^{M E}$ and is given by [11] and [12] as: 


$$
\begin{aligned}
& S_{i j}^{M E}=\int_{\Gamma_{M E}} \frac{\alpha_{i}^{M E}}{r} \frac{\alpha_{j}^{M E}}{n} \Gamma_{M E} \\
& S_{i j}^{M E}=\int_{0}^{b} \frac{\alpha_{i}^{M E}\left(r_{2}, z\right)}{r_{2}} \frac{\alpha_{j}^{M E}\left(r_{2}, z\right)}{\partial r} d z \\
& -\int_{0}^{b} \frac{\alpha_{i}^{M E}\left(r_{1}, z\right)}{r_{1}} \frac{\alpha_{j}^{M E}\left(r_{1}, z\right)}{\partial r} d z
\end{aligned}
$$

$r_{1}$ and $r_{2}$ are the boundaries of the Macro-Element and $b$ its length.

The variation of the potential magnetic vector versus time is solved in time-domain using constant time steps denoted by $\Delta t$, the derivative is determined as a mean value from two successive steps. When $A^{*^{t+\Delta t}}$ represents any variable at the current time step and $A^{*^{t}}$ is the same variable at the previous step, the derivatives are approximated by:

$$
\frac{\partial A^{*}}{\partial t}=\frac{A^{* t+\Delta t}-A^{* t}}{\Delta t}
$$

The algebraic system of the electromagnetic equation in term of magnetic vector potential and with taking into account the Macro-Element stiffness matrix, is given as fellow:

$$
\begin{aligned}
& \left(\frac{v}{4 r \Delta}\left(\begin{array}{lll}
S_{i i} & S_{i j} & S_{i k} \\
S_{j i} & S_{j j} & S_{j k} \\
S_{k i} & S_{k j} & S_{k k}
\end{array}\right)+\left(\begin{array}{ccc}
S_{i i}^{M E} & S_{i j}^{M E} & S_{i k}^{M E} \\
S_{j i}^{M E} & S_{j j}^{M E} & S_{j k}^{M E} \\
S_{k i}^{M E} & S_{k j}^{M E} & S_{k k}^{M E}
\end{array}\right)\right)\left\{\begin{array}{c}
A_{i}^{* t+\Delta t} \\
A_{j}^{* t+\Delta t} \\
A_{k}^{* t+\Delta t}
\end{array}\right\} \\
& +\frac{v}{4 r \Delta} \frac{1}{\Delta t}\left(\begin{array}{lll}
K_{i i} & K_{i j} & K_{i k} \\
K_{j i} & K_{j j} & K_{j k} \\
K_{k i} & K_{k j} & K_{k k}
\end{array}\right)\left\{\begin{array}{c}
A_{i}^{* t+\Delta t} \\
A_{j}^{* t+\Delta t} \\
A_{k}^{* t+\Delta t}
\end{array}\right\}=\frac{J_{s} \Delta}{3}\left\{\begin{array}{l}
1 \\
1 \\
1
\end{array}\right\}+\frac{v}{4 r \Delta} \frac{1}{\Delta t}\left(\begin{array}{lll}
K_{i i} & K_{i j} & K_{i k} \\
K_{j i} & K_{j j} & K_{j k} \\
K_{k i} & K_{k j} & K_{k k}
\end{array}\right)\left\{\begin{array}{c}
A_{i}^{* t} \\
A_{j}^{* t} \\
A_{k}^{* t}
\end{array}\right\} \\
& \left.+\frac{J_{s} \Delta}{3}\left\{\begin{array}{l}
1 \\
1 \\
1
\end{array}\right\}+\frac{v_{0}}{2}\left(\begin{array}{l}
b_{m z} \\
b_{j} \\
b_{k}
\end{array}\right)-b_{m r}\left(\begin{array}{l}
c_{i} \\
c_{j} \\
C_{k}
\end{array}\right)\right)
\end{aligned}
$$

Where:

$$
\begin{gathered}
\left\{\begin{array}{l}
S_{i j}^{M E} \neq 0 \text { on the nodes in the bounderies of the Macro Element } \\
S_{i j}^{M E}=0
\end{array}\right. \\
b_{i, j, k} \text { Elsewhere }
\end{gathered}
$$




\section{B. Electrical Circuit Model of the Electromagnetic Damper}

The equivalent electrical circuit of the Electromagnetic Damper is shown in Figure 3, where $R_{\text {coil }}$ and $L_{\text {coilare the resistance and inductance of the coil, and } R_{L o a d} \text { as the external }}$ resistance characterising the energy regeneration.

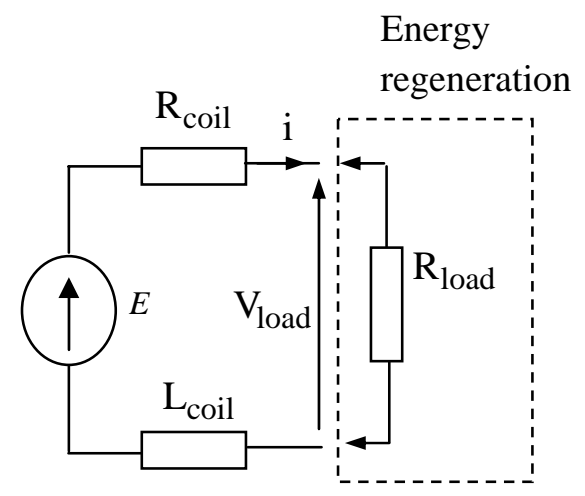

Figure 3. Electrical circuit model of the EEDR

The electrical load connected to the damper determines the relation ship between the circuit current and the induced electromotive force $E$. When the load is $R_{\text {load }}$, the circuit equation is:

$$
E=\left(R_{\text {coil }}+R_{\text {load }}\right) i+L_{\text {coil }} \frac{d i}{d t}
$$

Neglecting the coil's inductance, the maximum power loss is obtained when the regenerative energy is zero $\left(R_{\text {Load }}=0\right)$ i.e. the stator coils are short circuited. In addition, the maximum power regeneration occurs as the external resistance is set equal to the internal resistance. The power is given by the following equation:

$$
p_{e}=\frac{E^{2}}{R_{\text {coil }}+R_{\text {load }}}
$$

\section{Mechanical Equation}

The friction force of the electromagnetic damper can be neglected. Therefore, the dynamical equations of the suspension system (Figure 1) are given by (18) [7], [10] which can be written in state variable form as (19):

$$
\begin{aligned}
& m_{s} \ddot{z}_{s}=-k_{s}\left(z_{s}-z_{u}\right)-b_{s}\left(\dot{z}_{s}-\dot{z}_{u}\right)+F_{a} \\
& \boldsymbol{m}_{u} \ddot{z}_{u}=k_{s}\left(z_{s}-z_{u}\right)+b_{s}\left(\dot{z}_{s}-\dot{z}_{u}\right)-k_{u}\left(z_{u}-z_{r}\right)-F_{a}
\end{aligned}
$$

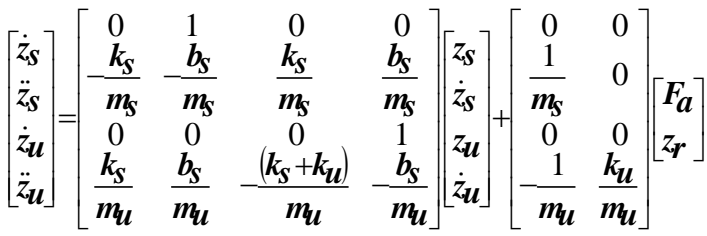

Where, $\dot{z}_{s}, \dot{z}_{u}$ and $\ddot{z}_{s}, \ddot{z}_{u}$ are the velocities and accelerations of sprung mass and unsprung mass respectively. $\left(z_{s}-z_{u}\right)$ and $\left(z_{u}-z_{r}\right)$ are the deflection of suspension and tyre respectively. 
The Lorentz low is used to calculate the force exerted on the mover due to current flowing through the coils. The magnetic force is given by:

$$
F_{a}=N_{a} \int i d \vec{l} \wedge \vec{B}_{r}
$$

Where, $d \vec{l}$ the tangential direction to the coil turns. Br is the magnetic flux density through the coil in the radial direction.

\section{Results and discussion}

Considering the axial symmetry of the EERD, the generator can be simplified to a 2D axial symmetry model in cylindrical coordinates. Only one fourth of the structure is studied.

The homogeneous Dirichlet condition of the magnetic vector potential is imposed at the boundary $\left(\Gamma-\Gamma_{M E}\right)$ and at the symmetry axis. Figure 4 shows the finite element mesh associated to the Macro-Element of the solved domain. The finite element is considered as a first order triangular element.

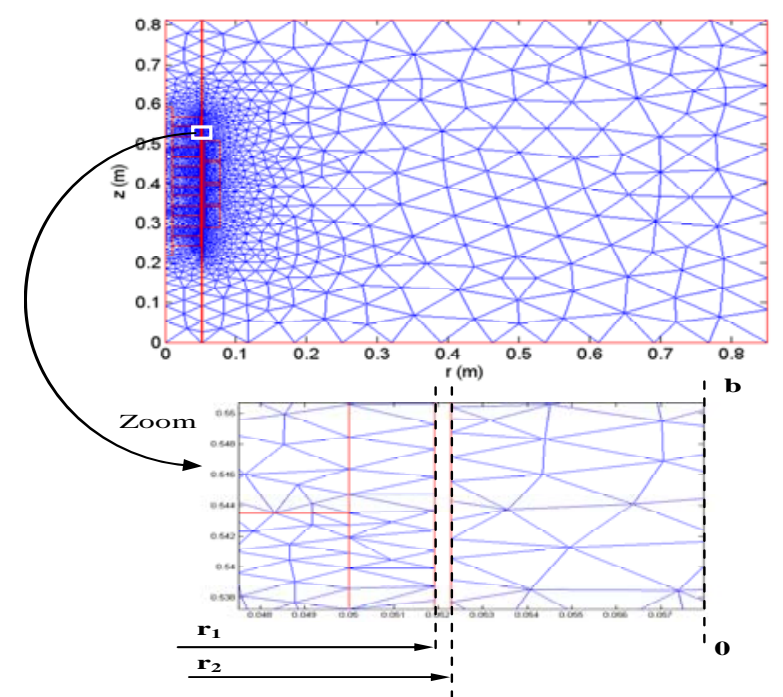

Figure 4. Finite element mesh associated to the Macro-Element of the studied domain

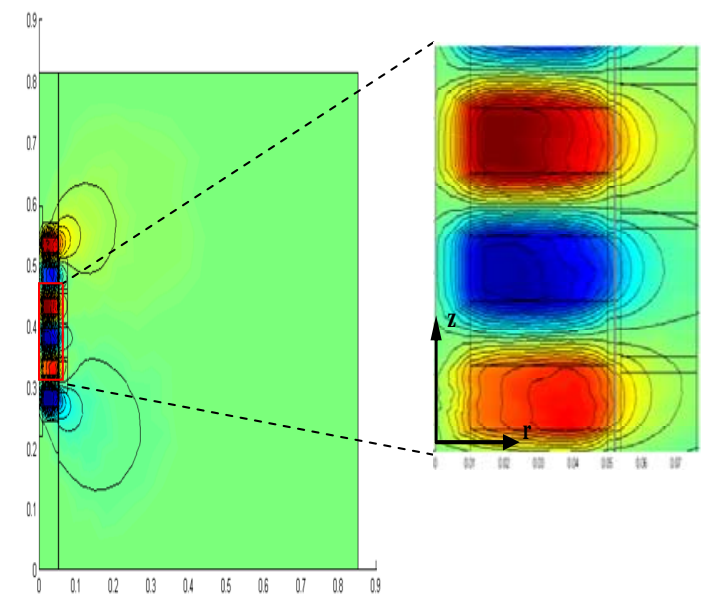

Figure 5. Magnetic flux line in the EERD 
Figure 5 shows the magnetic flux lines due to the magnetic excitation. The variation of both radial and axial flux densities with position along the translator is shown in Figure 6. Figure 7 and Figure 8 present comparison between the computed and the measured axial and radial flux densities at $3.8 \mathrm{~mm}$ above translator. Figure 8 shows the comparison between the computed and the measured radial flux density at $17.6 \mathrm{~mm}$ above the translator.

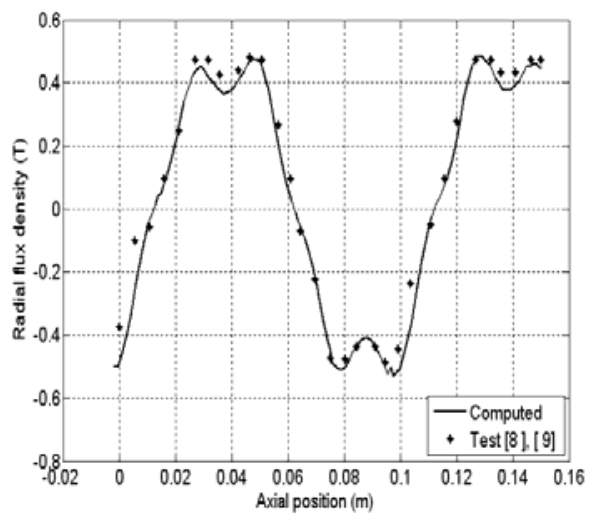

Figure 6. Comparison of computed and measured radial flux density at $3.8 \mathrm{~mm}$ above translator

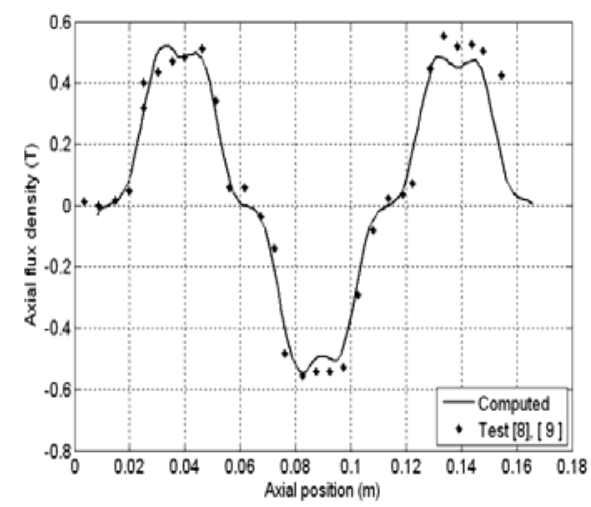

Figure 7. Comparison of computed and measured axial flux density at $3.8 \mathrm{~mm}$ above translator

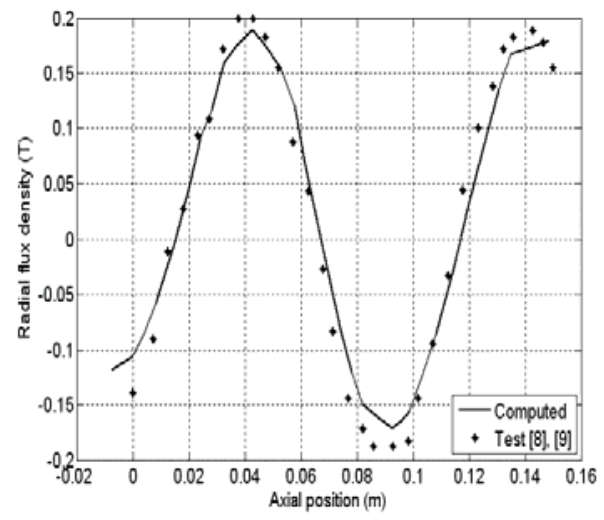

Figure 8. Comparison of computed and measured Axial flux density at $17.6 \mathrm{~mm}$ above translator 


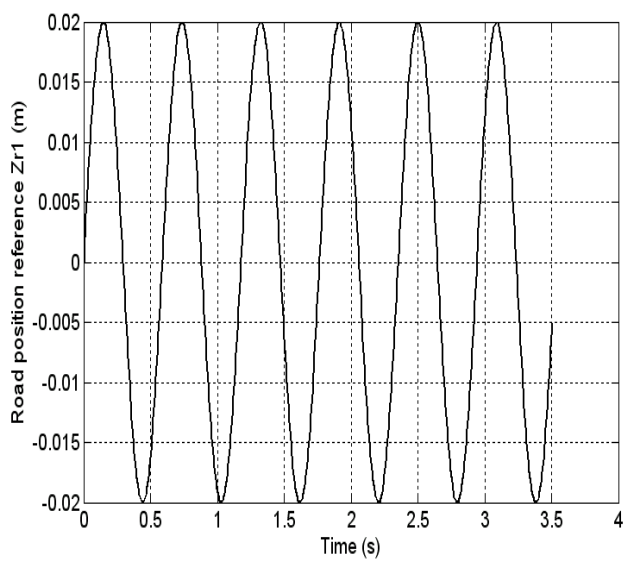

Figure 9. Road position reference versus time (First cycle of vibrations)

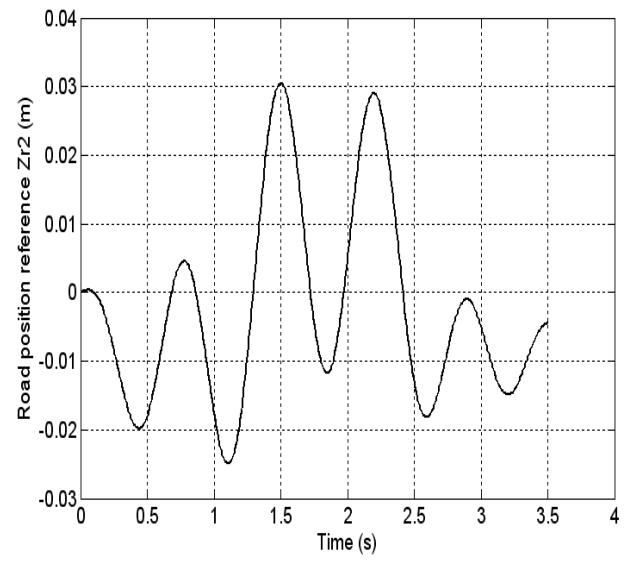

Figure 10. Road position reference versus time (Second cycle of vibrations)

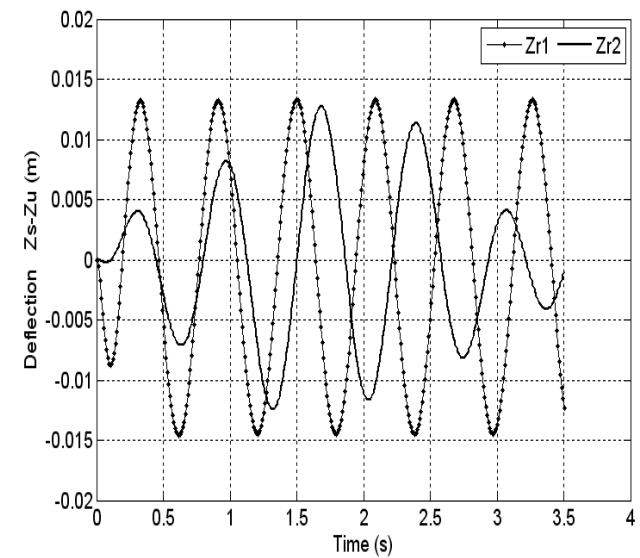

Figure 11. Deflection versus time for the first and the second cycle of vibrations 
The developed model is applied to study and to analyse the Electromagnetic Energy Recovery Damper. The permanent magnet is of $\mathrm{NdFeB}$, its remnant flux density is $1.2 \mathrm{~T}$ and the relative permeability is unity. The steel magnetic permeability is considered as infinite [8].Figure 9 and Figure10 show the assumed cycles of vibrations. These vibrations represent the input-road irregularities reference position $Z_{r}$ versus time imposed to quantify the corresponding maximum output electrical power regeneration. The first cycle of vibrations has sinusoidal waveform with maximum amplitude of $0.02 \mathrm{~m}$ and the second has an arbitrary waveform with maximum amplitude of $0.03 \mathrm{~m}$.

Figure11 presents the deflection representing the differential position between the sprung and the unsprung mass for the considered cycle of vibrations. Figure 12 shows the magnetic force versus time corresponding to respectively the first and second cycle of vibrations. From the graphic, the magnetic force caused by the first cycle is larger compared to the second cycle.

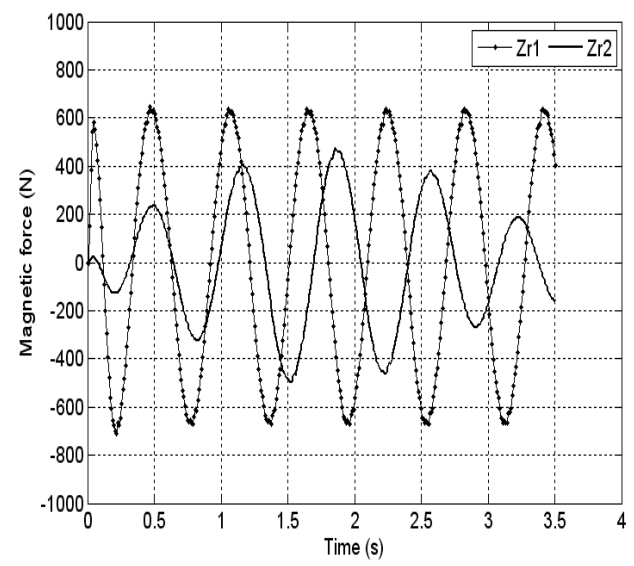

Figure 12. Magnetic force versus time for the first and the second cycle of vibration

Figure 13 gives the induced electromotive force versus time corresponding to the first and second cycles of vibrations. The electromotive force depends of the relative velocity between the coil and the translator and corresponding to the differential velocity between the sprung and the unsprung mass. The amplitude of the total induced electromotive force is of $46.93 \mathrm{~V}$ for the first cycle of vibrations and is of $34.25 \mathrm{~V}$ for second cycle.

Figure 14 gives the current versus time corresponding to the first and second cycle of vibrations when the load is a resistance $R_{\text {load }}=R_{\text {coil }}$. The amplitude of the total current is of $0.93 \mathrm{~A}$ for the first cycle of vibrations and is of $0.68 \mathrm{~A}$ for the second cycle.

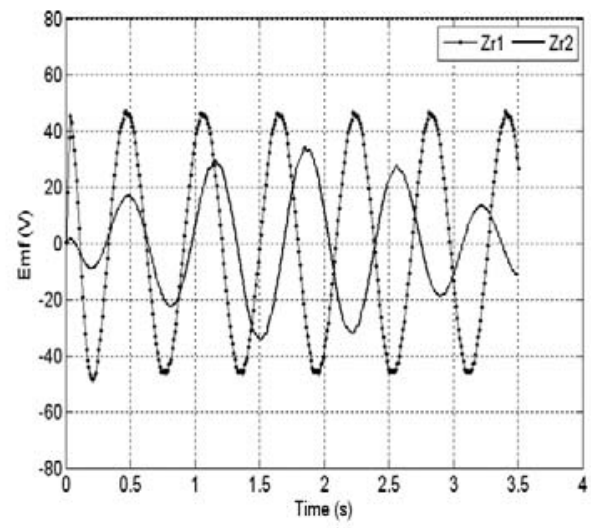

Figure 13. Induced electromotive force versus time for the first and second cycle of vibrations 


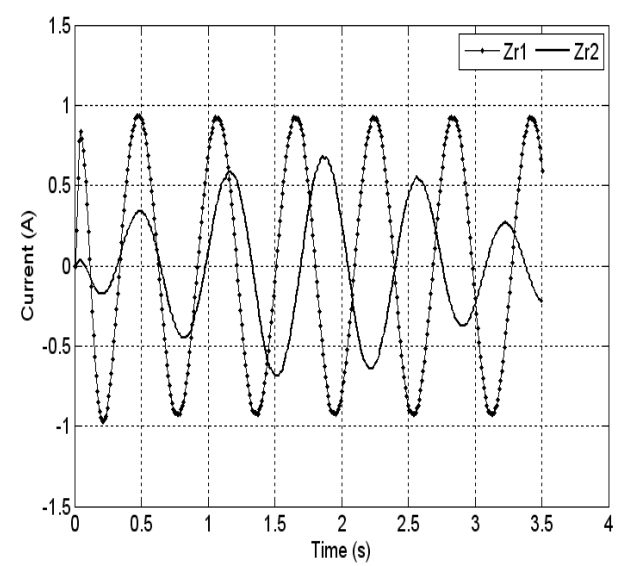

Figure 14. Current versus time for the first and second cycle of vibrations

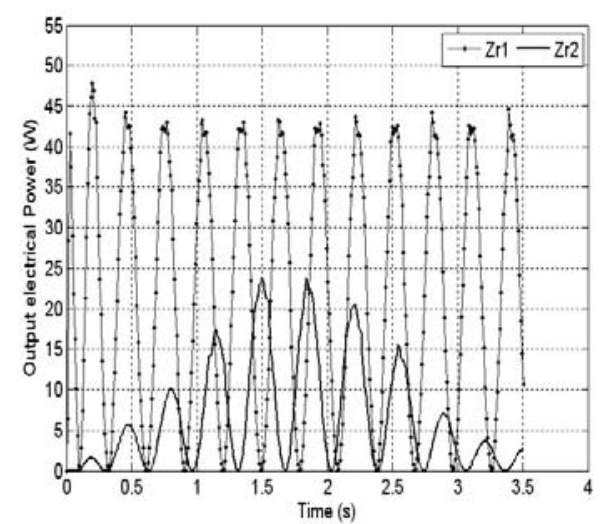

Figure 15. Maximum output electrical power versus time for the first and second cycle of vibrations

Based on expression (17) and assuming $R_{\text {load }}=R_{\text {coil }}$, the maximum output electrical power versus time is obtained and shown in Figure15. The peak value of electrical power is $47.82 \mathrm{~W}$ corresponding to the first cycle of vibrations and is of $23.93 \mathrm{~W}$ for the second cycle. So, the average output electrical power was $22.13 \mathrm{~W}$ and $6.36 \mathrm{~W}$ respectively for the first cycle of vibrations and the second.

\section{Conclusion}

In this paper an electromagnetic energy recovery damper (EERD) for road vehicles has been presented. An electromagnetic-mechanical model is developed and implemented under Matlab and then applied to analyse the electromagnetic energy recovery damper. Through this model a quantification of the maximum output electrical power level for imposed various cycles of vibrations representing the roads irregularities is obtained. We notice that the level of the electrical power depends on the cycle of vibration wave forms and its amplitude. The electromagnetic energy recovery damper can be used as generator to improve the power and the efficiency of electrical vehicles.

\section{References}

[1] W. C. Kruckemeyer, H. C. Buchanan, Jr., and W. V. Fannin, "Rotational actuator for vehicle suspension damper, “U.S. Patent 4644 200, Feb. 17, 1987. 
[2] J. H. Beno, D. A. Weeks, and W. F. Weldon, “Constant force suspension, near constant force suspension, and associated control algorithms,” U.S. Patent 5999 868, Dec. 7, 1999.

[3] D. A. Weeks, D. A. Bresie, J. H. Beno, and A. M. Guenin, "The design of an electromagnetic linear actuator for an active suspension,” in Proc. SAE Steering and Suspension Technology Symp., Detroit, MI, 1999, pp. 1-11.

[4] I. Martins, J. Esteves, F. P. da Silva, and A. Torn2, “Automobile suspensions using electromagnetic linear actuators," in Proc. IFAC Conf. Mechatronics, Darmstadt, Germany, 2001, pp. 203-208.

[5] B. Lequesne, "Permanent magnet linear motors for short strokes," IEEE Trans. Ind. Applicat., vol. 32, no. 1, pp. 161-168, Jan./Feb. 1996.

[6] B. V. Murty and R. R. Henry, "Active vehicle suspension with brushless dynamoelectric Actuator,” U.S. Patent 5091 679, Feb. 25, 1992.

[7] Ismenio Martins, Jorge Esteves, Gil D. Marques, and Fernando Pinada Silva, "PermanentMagnets Linear Actuators Applicability in Automobile Active Suspensions”, IEEE transactions On Vehicular Technology, Vol.55, N¹, pp.86-94., January 2006.

[8] N.J. Baker, "Linear Generators for Direct Drive Marine Renewable Energy Converters”, PHD. Thesis, University of Durham, July 2003, Uk.

[9] N. J. Baker , M. A. Mueller, \& E. Spooner, "Permanent Magnet Air-Cored Tubular Linear Generator For Marine Energy Converters”, Second International Conference on Power Electronics, Machines and Drives, PEMD 2004 (Conf. Publ. No. 498) Vol.2, pp. $862-867$.

[10] M. Chaves, J. Maia, J.Esteves, “Analysis of an Electromagnetic Automobile Suspension System” in Proc. ICEM08, Portugal, 2008, Paper ID 1285.

[11] H.Mohellebi, M.E.Latrèche, and M.Féliachi, "Coupled axisymmetric analytical and finite element analysis of induction devices having moving parts”, IEEE Tran. Magn. vol.34, no. 5 pp.3308-3310, Sept. 1998.

[12] F.Azzouz, B.Bendjima, M.Féliachi, “Application of Macro-Element and Finite Element Coupling for the Behaviors Analysis of Magnetoforming Systems”, IEEE Tran. Magn. vol.35, no.3 pp.1845-1848, May.1999.

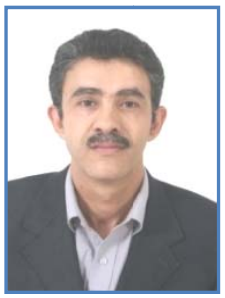

Mustapha Zaouia was born in Tizi-Ouzou, Algeria. He received Magister degree from Polytechnic School, Algiers, Algeria in 2001and the PhD degree in the Mouloud Mammeri University of Tizi-Ouzou, Algeria. He is Assistant Professor. His research interests the electromagnetic-mechanical modelling, numerical simulation of electromagnetic machines particularly the linear machines and permanent-magnet machines applied to the electrical and hybrid vehicles.

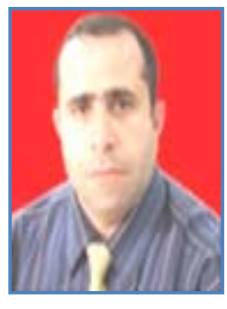

Hakim Denoun was born in Algiers, Algeria. He received his Bsc degree in electrical engineering from the Mouloud Mammeri University, Tizi-Ouzou, Algeria, and the D.E.A degree from Paris 6, France and the Magister degree from Polytechnic School, Algiers, Algeria in 1998 and 2001 respectively. He got his $\mathrm{PhD}$ degree in Electrical Engineering from The University of Mouloud Mammeri University, Tizi-Ouzou .He is currently a senior lecturer at the same university. His research interests include electrical machines and drives, power electronics and control systems. 


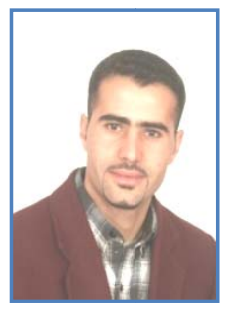

Nabil Benyahia received the degree in electrical engineering and the Magister degree from the Bejaia university, Algeria in 2002 and 2005 respectively. Presently he prepares the $\mathrm{PhD}$ degree in the Bejaia University, Algeria and he is Assistant-Professor in the Electrical Engineering Department, University of Tizi-Ouzou, Algeria. His research interests the fuel cell modelling and numerical simulation of renewable energy particularly the photovoltaic energy, wind energy and hydrogen energy.

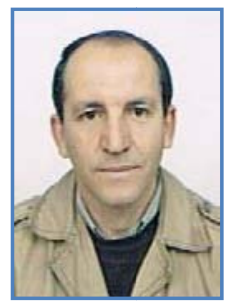

Mustapha Bouheraoua was born in Tizi-Ouzou; he received Magister degree and $\mathrm{PhD}$ degree in 2008 in Electrical Engineering respectively in 2001 and 2008 from Mouloud Mammeri University of Tizi-Ouzou, Algeria. His research interests include electrical and thermal models of machines, power electronics and control systems.

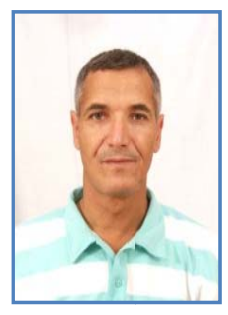

Nacereddine Benamrouche received his $\mathrm{PhD}$ in Electrical Engineering from The University of Sheffield, U.K. He worked as teaching assistant at the University of Leeds in 1990/1991, and as a Head of Department in Najran Technical College of Technology, Saudi Arabia, from 2000-2004. He is currently a professor at the Electrical Engineering Department, University of Tizi-Ouzou, Algeria, and occupies the chair of Vice Chancellor to postgraduate studies and research at the same University. His research interests include electrical machines and drives, power electronics and control systems, and renewable energy. 